\title{
Severe catheter kinking and entrapment during transbrachial angiography: percutaneous retrieval with a slender approach
}

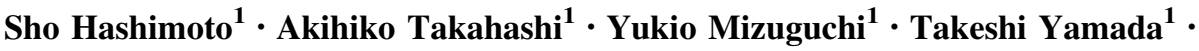 \\ Norimasa Taniguchi ${ }^{1} \cdot$ Tetsuya Hata $^{1}$
}

Received: 12 December 2015/Accepted: 1 March 2016/Published online: 11 March 2016

(c) The Author(s) 2016. This article is published with open access at Springerlink.com

\begin{abstract}
A 74-year-old man previously treated with bilateral femoro-popliteal bypass was referred to our hospital for the treatment of a severely tangled 4-Fr diagnostic catheter in the right brachial artery. We inserted a 5-Fr sheath introducer from the proximal right femoral artery and advanced a 5-Fr JR catheter to the right brachial artery. A Sion coronary guidewire was then advanced for the tip of the tangled catheter, and a 4-mm gooseneck snare catheter was inserted through the guidewire. The tip of the catheter was caught and successfully removed after clockwise untwisting. The patient was discharged the next day without any complications.
\end{abstract}

Keywords Snare $\cdot$ Kinked catheter $\cdot$ Entrapped $\cdot$ Slender approach

\section{Introduction}

Kinking of the catheter shaft sometimes occurs during diagnostic and interventional catheterization after excessive twisting. Usually, the kinking of the shaft can be released with counter rotation of the catheter and the damaged catheter can be retrieved safely [1]. However,

Akihiko Takahashi

a-takahashi@wine.ocn.ne.jp

1 Department of Cardiology, Sakurakai Takahashi Hospital, 5-18-1 Oikecho, Sumaku, Kobe, Hyogo 654-0026, Japan for cases with excessive kinking, when this manipulation fails, further invasive procedures should be considered.

In this report, we present successful retrieval of a severely deformed catheter with multiple kinking that was stuck in the right brachial artery with a less invasive procedure from the right femoral artery where femoro-popliteal bypass was placed.

\section{Case report}

A 74-year-old man with previous medical history of bilateral femoro-popliteal bypass was referred to our hospital for the retrieval of a kinked catheter in the right brachial artery, which occurred during transbrachial angiography for the left carotid artery with a 4-Fr Simmons catheter after excessive rotation of the catheter to obtain the optimum direction of the tip of the catheter without pressure monitoring. The $\mathrm{X}$-ray fluoroscopic view showed the entrapped catheter with multiple fractures in the mid right brachial artery (Fig. 1). To try to retrieve it, we added gentle rotation and pull for the catheter; however, the kinking was not released. We inserted a 5-Fr sheath introducer (Terumo, Tokyo, Japan) from the right femoral artery just above the anastomosis site of the PTFE graft and advanced a 5-Fr JR 3.5 guiding catheter (Heartrail II, Terumo, Tokyo, Japan) to the proximal right brachial artery. Then, we advanced a Sion coronary guide wire to the tip of the tangled catheter and inserted a 4-mm gooseneck snare catheter (ev3 Inc. Plymouth, MN, USA) through the guidewire, and caught and fixed the tip of the tangled diagnostic catheter (Fig. 2). We added counterclockwise rotation and pull to the catheter shaft (Fig. 3a-c). Finally, we 


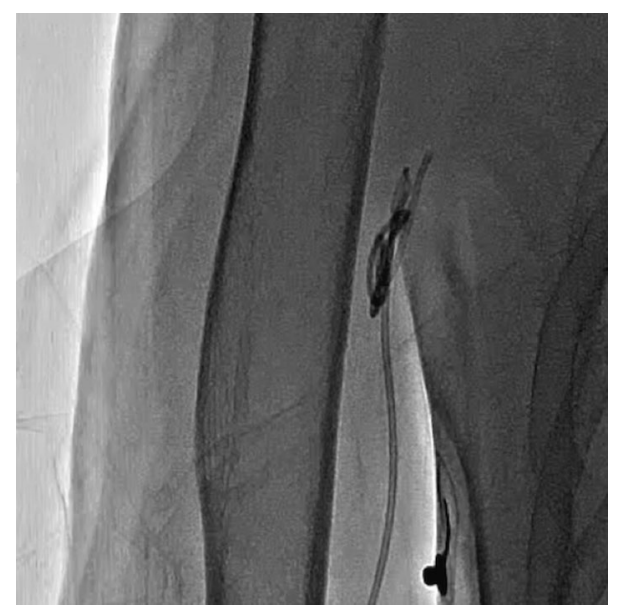

Fig. 1 The X-ray fluoroscopic view showing the entrapped catheter with multiple fractures in the mid right brachial artery

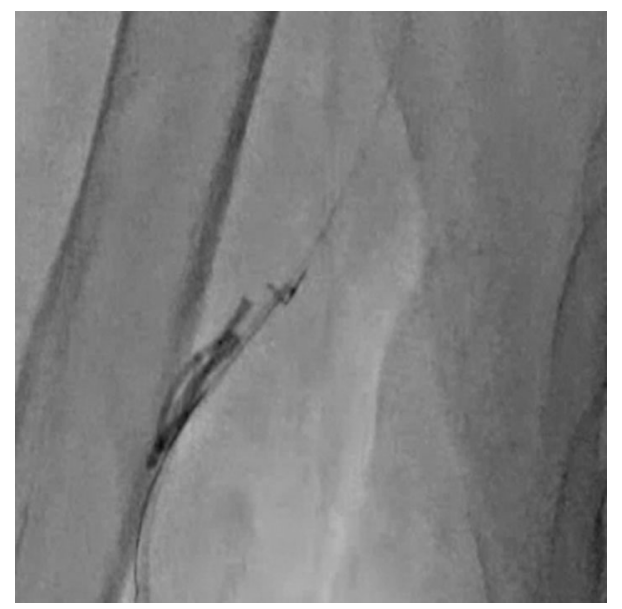

Fig. 2 A 4-mm gooseneck snare catheter through the 5-Fr JR 3.5 guide catheter inserted from the right femoral artery, caught and fixed the tip of the kinked diagnostic catheter

successfully retrieved the catheter from the sheath introducer in the brachial artery. The patient was discharged the next day without any sequelae.

\section{Discussion}

We demonstrate a case with a severely kinked diagnostic catheter from carotid artery angiography that was successfully treated with a 4-mm gooseneck snare through a 5-Fr guiding catheter inserted from the right femoral artery, where the anastomosis of the femoro-popliteal bypass with PTFE graft was placed. The catheter developed kinking at 8 points under the specific condition in which the arterial pressure was not monitored, unlike standard cardiac catheterization where cardiologists usually can notice such kinking with pressure monitoring. To the best of our knowledge, this is the worst case among the recent case reports of catheter kinking.

If the kinking cannot be released with conventional counter rotation of the catheter, several procedures including the use of external compression to fix the distal part of the kinked catheter [2]; use of a larger long sheath with a conventional guidewire [3, 4]; and use of a balloon catheter or vascular snare to fix the catheter $[5$, 6] may be considered. In this case, fixation with either balloon catheter or snare is considered the only solution because of the complexity of the deformation. In the current case, we initially tried to put the Sion wire into the tip of the kinked catheter to fix the catheter from inside with a balloon catheter, because this is less expensive than the snare.

We used a 5-Fr guiding catheter to avoid further vascular complication from the femoral artery in which bypass graft was anastomosed. A 5-Fr system proved to have enough inner lumen for a 4-mm gooseneck snare with a 0.014-in. coronary guidewire, although recent reports suggested the use of 7-Fr or larger-sized guiding catheters for retrieval with snare. Further, deployment of a 4-mmsized snare in the brachial artery provided instant trapping of the catheter tip with aid of the guidewire; however, when it came to the larger artery including the subclavian artery or thoracic aorta, a 4-mm snare is considered too small for trapping a catheter tip effectively. 
Fig. 3 After fixing the tip of the kinked catheter with a gooseneck snare (a), counter clockwise rotation was add to the catheter shaft (b). This manoeuver lead to the release of the kinking of the catheter and successful retrieval (c)
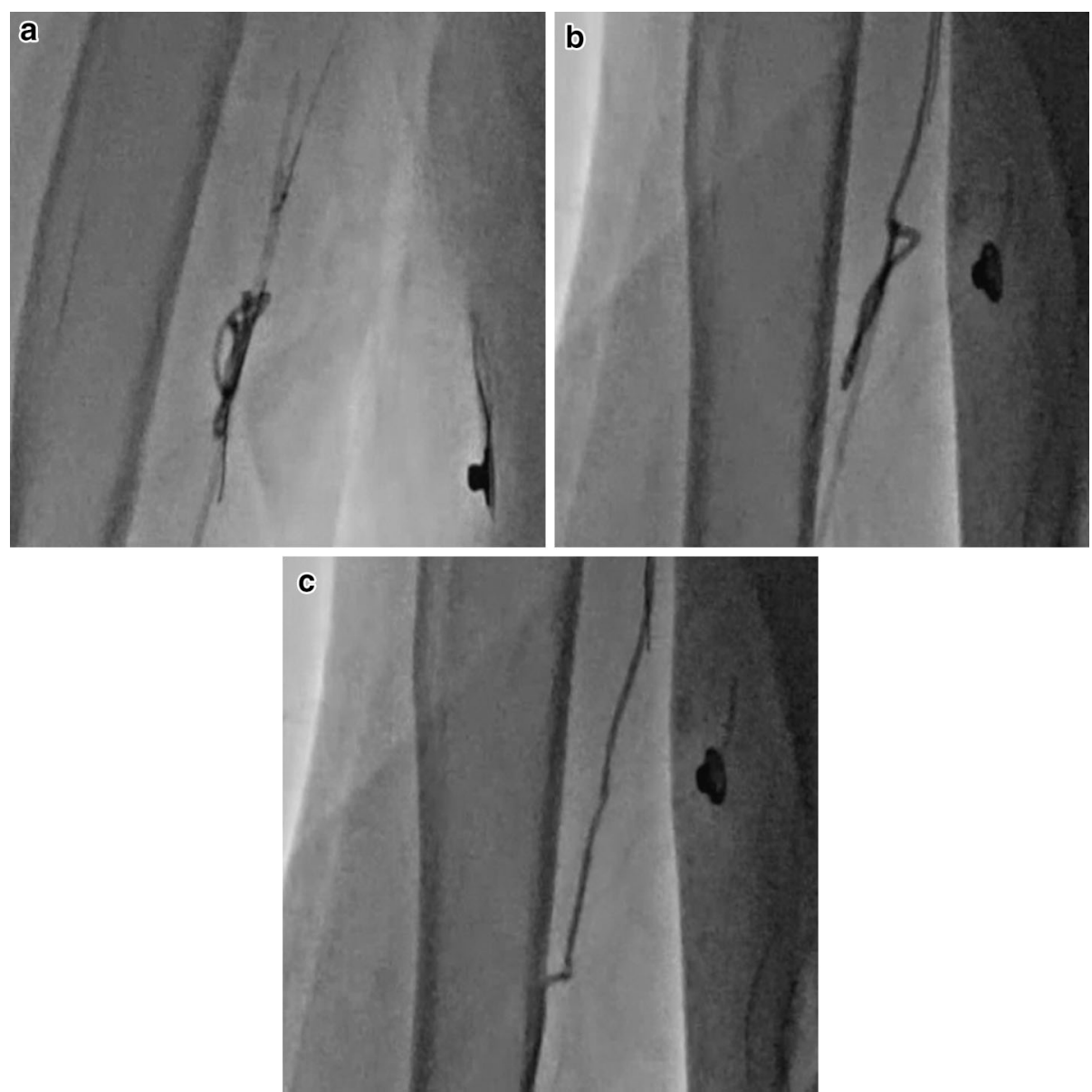

\section{Conclusion}

We considered that this approach with a smaller (5-Fr) catheter is feasible and effective for the retrieval of the kinked guide catheter and may contribute to the reduction of further vascular complications, particularly in patients with a diseased approach site.

\section{Compliance with ethical standards}

Conflict of interest The authors have no conflict of interest. The study was performed in accordance with the Helsinki Declaration, and the patient gave his written informed consent. The study protocol was approved the Sakurakai Takahashi Hospital Institutional Review Board for potential publication in Cardiovascular Intervention and Therapeutics.

Open Access This article is distributed under the terms of the Creative Commons Attribution 4.0 International License (http://crea tivecommons.org/licenses/by/4.0/), which permits unrestricted use, distribution, and reproduction in any medium, provided you give appropriate credit to the original author(s) and the source, provide a link to the Creative Commons license, and indicate if changes were made.

\section{References}

1. Aminian A, Fraser DG, Dolatabadi D. Severe catheter kinking and entrapment during transradial coronary angiography: percutaneous retrieval using a sheathless guide catheter. Catheter Cardiovasc Interv. 2015;85(1):91-4.

2. Patel T, Shah S, Pancholy S. A simple approach for the reduction of knotted coronary catheter in the radial artery during the transradial approach. J Invasive Cardiol. 2011;23(5):E126-7.

3. Waked A, Khoueiry G, Bhat T. Entrapment of a looped/kinked catheter in the brachial artery and its successful retrieval during transradial coronary catheterization. J Invasive Cardiol. 2012;24(9):471-2.

4. Leibundgut G, Loffelhardt N, Neumann FJ. Percutaneous retrieval of a twisted guide catheter using a longer second radial sheath. Catheter Cardiovasc Interv. 2014;83(4):560-3.

5. Michael TT, Banerjee S, Brilakis ES. Percutaneous retrieval of a fractured guide catheter using contralateral snaring. J Invasive Cardiol. 2012;24(8):E176-8.

6. Layland J, McGeoch R, Sood A. Novel method of rescuing kinked guide catheter from axillary artery in transradial coronary intervention: the balloon retrieval technique. J Invasive Cardiol. 2012;24(9):E205-6. 